\title{
COBORDISM AND THE NONFINITE HOMOTOPY TYPE OF SOME DIFFEOMORPHISM GROUPS
}

\author{
DANIEL S. CHESS
}

\begin{abstract}
Unoriented cobordism, a geometric construction, and a theorem of Browder on finite $H$-spaces are used to give new examples of manifolds whose diffeomorphism groups have identity component of nonfinite homotopy type.
\end{abstract}

The nature of the group $\operatorname{Diff}(M)$ of smooth diffeomorphisms of a smooth manifold is of considerable current interest. It is known in many cases, and expected for most $M$, that $\operatorname{Diff}_{0}(M)$, the identity component of $\operatorname{Diff}(M)$ under the $C^{\infty}$ topology, is not of finite homotopy type [1]. Our purpose is to give a simple construction of examples of this phenomenon.

The existence of these examples follows directly from a result in the theory of $H$-spaces, a geometrical construction, and a calculation in the unoriented cobordism ring.

FACT 1. Let $X$ be an arcwise connected $H$-space. Then if $X$ is of finite homotopy type, $\pi_{2}(X)=0[2]$.

Let $\mathcal{R}_{*}$ denote the unoriented cobordism ring. An element $[M]$ of $\Re_{*}$ is said to fiber over the $n$-sphere $S^{n}$ if and only if there is a representative $M$ of $[M]$ and a smooth fiber bundle $p: M \rightarrow S^{n}$. Denote by $\Re^{n}$ the subset of elements of $\Re_{*}$ which fiber over $S^{n}$. Clearly $R^{n}$ is an ideal of $\Re_{*}$.

FACT 2. $\Re^{n+1} \subset \Re^{n}$.

The proof is by the following construction due to $\mathrm{H}$. Winkelnkemper. Suppose $[M] \in \mathscr{R}^{n+1}$ is represented by $F \stackrel{i}{\rightarrow} M \stackrel{p}{\rightarrow} S^{n+1}$. As $0=\left[F \times S^{n+1}\right] \in \mathcal{R}^{n+1}$ by considering $M+F \times S^{n+1}$ (disjoint union), $M$ is represented by

$$
F \times\{-1,1\} \rightarrow M+F \times S^{n+1} \rightarrow S^{n+1},
$$

where

$$
F \times\{-1,1\}=\partial(F \times[-1,1])
$$

Now

$$
\begin{gathered}
M+F \times S^{n+1}=F \times\{-1,1\} \times D^{n+1} \cup F \times\{-1,1\} \times D^{n+1} \\
\left(F \times\{-1,1\} \times S^{n}, \tilde{g}\right),
\end{gathered}
$$

where $g:\left(S^{n}, *\right) \rightarrow(\operatorname{Diff}(F)$, id $)$ is a smooth map and $\tilde{g}: F \times\{-1,1\} \times S^{n} \rightarrow F \times$ $\{-1,1\} \times S^{n}$ is given by $\tilde{g}(x,-1, s)=(g(s)(x),-1, s)$ and $\tilde{g}(x, 1, s)=(x, 1, s)$.

Received by the editors September 13, 1982 and, in revised form, May 2, 1983.

1980 Mathematics Subject Classification. Primary 57R50, 57R75.

(C)1983 American Mathematical Society $0002-9939 / 83 \$ 1.00+\$ .25$ per page 
Extend $\tilde{g}$ to $\bar{g}$ on $F \times\left(\left[-1,-\frac{1}{2}\right] \cup\left[\frac{1}{2}, 1\right]\right) \times S^{n}$ by $\bar{g}\left(x,-t^{2}, s\right)=\left(g(s)(x),-t^{2}, s\right)$ and $\bar{g}\left(x, t^{2}, s\right)=\left(x, t^{2}, s\right)$. The manifold

$$
\begin{aligned}
N= & F \times[-1,1] \times D^{n+1} \cup F \times[-1,1] \times D^{n+1} \\
& \left(F \times\left(\left[-1,-\frac{1}{2}\right] \cup\left[\frac{1}{2}, 1\right]\right) \times S^{n}, \bar{g}\right)
\end{aligned}
$$

whose boundary is the disjoint union of $M \cup F \times S^{n+1}$ and a fiber bundle over $S^{n}$ with fiber

$$
\begin{gathered}
F \times\left[-\frac{1}{2}, \frac{1}{2}\right] \cup F \times\left[-\frac{1}{2}, \frac{1}{2}\right]=F \times S^{1} \\
\left(\partial\left(F \times\left[-\frac{1}{2}, \frac{1}{2}\right]\right), \text { id }\right)
\end{gathered}
$$

is the required cobordism.

We now describe the relation between the clutching functions for the boundary of $N$. Denote by $\Omega_{s}(\operatorname{Diff}(F))$ the appropriately topologized space of smooth maps of $\left(S^{1}, *\right)$ to $(\operatorname{Diff}(F)$, id $)$. Then there is an obvious map

$$
e: \Omega_{s}(\operatorname{Diff}(F)) \rightarrow \operatorname{Diff}\left(F \times S^{1}\right)
$$

given by $e(l)(x, t)=(l(t)(x), t)$. Denoting by $\Omega(\operatorname{Diff}(F))$ the loop space of $\operatorname{Diff}_{0}(F), \quad i: \Omega_{s}(\operatorname{Diff}(F)) \rightarrow \Omega(\operatorname{Diff}(F))$ is a homotopy equivalence. Regarding $\pi_{i}\left(\operatorname{Diff}_{0}(F)\right)$ as $\pi_{i-1} \Omega_{s}(\operatorname{Diff}(F))$, we have the map

$$
\tilde{e}: \pi_{i}\left(\operatorname{Diff}_{0}(F)\right) \rightarrow \pi_{i-1}\left(\Omega_{s} \operatorname{Diff}(F)\right) \stackrel{e_{*}}{\rightarrow} \pi_{i-1} \operatorname{Diff}\left(F \times S^{1}\right) .
$$

Given a smooth fiber bundle $F \rightarrow M \rightarrow S^{n+1}, n \geqslant 1$, determined by a clutching class $g \in \pi_{n}\left(\operatorname{Diff}_{0}(F)\right)$, the bundle over $S^{n}$ constructed above has fiber $F \times S^{1}$ and clutching class $\tilde{e}(g) \in \pi_{n-1} \operatorname{Diff}\left(F \times S^{1}\right)$.

OBSERVATION 3. Let $0 \neq[M] \in \mathrm{oR}^{3}$ and let $F \rightarrow M \rightarrow S^{3}$ be a representative of $M$ with clutching class $g$. Then $0 \neq[g] \in \pi_{2} \operatorname{Diff}_{0}(F)$.

Thus in order to show the existence of manifolds $F$ with $\operatorname{Diff}_{0}(F)$ of nonfinite homotopy type, it suffices to show $0 \neq \Re^{i}$ for some $i \geqslant 3$.

As $\stackrel{R}{R}_{*}$ is a polynomial ring over $Z_{2}, \phi^{2}: \mathscr{R}_{*} \rightarrow \mathscr{R}_{*} ; \phi^{2}: x \mapsto x^{4}$ is an injective homomorphism. Let $K_{1} \subset \mathscr{R}_{*}$ be the kernel of $\chi: \mathscr{R}_{*} \rightarrow Z_{2}$, where $\chi$ is the mod 2 Euler characteristic.

FACT 4. $\phi^{2}\left(K_{1}\right) \subset \mathscr{R}{ }^{4}[3,7.3]$. Hence $0 \neq \Re^{4} \subset \Re^{3}$.

The generators of $\phi^{2}\left[K_{1}\right]$ represented by elements of $\Re^{4}$ which are given in $\S 7$ of [3] are all determined by $S^{3}=\mathrm{Sp}(1)$ actions on manifolds with evidently nontrivial rational pontryagin classes so that these examples differ from those of [1].

It is worthwhile to consider the examples of [3] more explicitly. Let $\mathbf{F}$ be $\mathbf{R}, \mathbf{C}$ or $\mathbf{H}$, let $G(\mathbf{F})$ be the group of unit norm elements of $F$, and let $S(k \mathbf{F})$ denote the unit sphere in $k \mathbf{F} . G(\mathbf{F})^{k+1}$ denotes the $(k+1)$-fold direct product of $G(\mathbf{F})$, and $\Sigma^{k}(\mathbf{F})$ denotes the $k$-fold direct product of $S(2 \mathbf{F})$. We define a $G(\mathbf{F})^{k+1}$ action on $\Sigma^{k}(\mathbf{F}) \times$ $S((n+1) \mathbf{F})$ by

$$
\begin{aligned}
\left(t_{1}, \ldots, t_{k+1}\right)\left(\left(q_{1}, p_{1}\right), \ldots,\left(q_{k}, p_{k}\right),\left(\rho_{1}, \ldots, \rho_{n+1}\right)\right) \\
=\left(\left(q_{1} t_{1}^{-1}, p_{1} t_{1}^{-1}\right), \ldots,\left(q_{j} t_{j}^{-1}, t_{j-1} p_{j} t_{j}^{-1}\right), \ldots,\left(q_{k} t_{k}^{-1}, t_{k-1} p_{k} t_{k}^{-1}\right)\right) \\
\quad\left(t_{k} \rho_{1} t_{k+1}^{-1}, \rho_{2} t_{k+1}^{-1}, \ldots, \rho_{n+1} t_{k+1}^{-1}\right)
\end{aligned}
$$


Denote the quotient manifold of this principal action by $V(n, k ; \mathbf{F})$. A point in the $p(n+k)$ manifold $V(n, k ; \mathbf{F}) \quad(p=1,2,4)$ is denoted $\left[\left(q_{1}, p_{1}\right), \ldots,\left(q_{k}, p_{k}\right)\right.$, $\left.\left(p_{1}, \ldots, p_{n+1}\right)\right]$. The map $V(n, k ; \mathbf{F}) \rightarrow \mathbf{F} P(1)=S^{1}, S^{2}, S^{4}$ given by

$$
\left[\left(q_{1}, p_{1}\right), \ldots,\left(q_{k}, p_{k}\right) ;\left(p_{1}, \ldots, p_{n+1}\right)\right] \rightarrow\left[\left(p_{1}, q_{1}\right)\right]
$$

is a fiber map with fiber $V(n, k-1 ; \mathbf{F})$ and structure group $G(\mathbf{F})$ where the action of $G(\mathbf{F})$ on $V(n, k-1 ; \mathbf{F})$ is given by

$$
\begin{aligned}
& t\left[\left(q_{1}, p_{1}\right), \ldots,\left(q_{k-1}, p_{k-1}\right),\left(\rho_{1}, \ldots, \rho_{n+1}\right)\right] \\
& \quad=\left[\left(q_{1}, t p_{1}\right),\left(q_{2}, p_{2}\right), \ldots,\left(q_{k-1}, p_{k-1}\right),\left(\rho_{1}, \ldots, \rho_{n+1}\right)\right] .
\end{aligned}
$$

Connor and Floyd established the following results, where $[M]$ denotes the class of $M$ in $\stackrel{R}{*}_{*}$.

TheOREM. $[V(n, k ; \mathbf{C})]=[V(n, k ; \mathbf{R})]^{2},[V(n, k ; \mathbf{Q})]=[V(n, k ; \mathbf{C})]^{2},[V(2,2 p ; \mathbf{R})]$ is indecomposable in $R_{2(p+1)}$.

As a straightforward result of Wall's computation of $\Omega_{*}$, oriented bordism [4] we have

Proposition. Let $F: \Omega_{*} \rightarrow \mathscr{R}_{*}$ be the forgetful map. If $x \in \mathcal{R}_{*}$ is a power of an even indecomposable then $x \notin F\left(\right.$ tor $\Omega_{*}$ ).

The manifold $V(n, k ; \mathbf{H})$ is orientable; choosing an orientation, let $\{V(n, k ; \mathbf{H})\}$ denote its class in $\Omega_{*}$. From the results just quoted we have $\{V(2,2 p ; \mathbf{H})\}$ is not torsion. Note that the Winkelnkemper construction produces oriented bordisms from oriented bundles. Also note that for oriented manifolds $F^{k}$ the map

$$
\pi_{i} \operatorname{Diff}_{0}(F) \rightarrow \pi_{i+1} \mathrm{~B} \mathrm{Diff}_{0}(F) \rightarrow \Omega_{i+k+1}
$$

that takes a fiber bundle over $S^{i+1}$ to the oriented cobordism class of its total space is a homomorphism. Thus the elements of $\pi_{2} \operatorname{Diff}_{0}\left(V(2,2(p-1) ; \mathbf{H}) \times S^{1}\right)$ produced by the Wilkelnkemper construction on $V(2,2 p ; \mathbf{H}) \rightarrow S^{4}$ are not torsion. We may then appeal to the classical theorem of Hopf, rather than that of Browder, in asserting the nonfinite homotopy type of $\operatorname{Diff}_{0}\left(V(2,2(p-1) ; \mathbf{H}) \times S^{1}\right)$.

\section{BIBLIOGRAPHY}

1. P. L. Antonelli, D. Burghelea and P. J. Kahn, The nonfinite homotopy type of some diffeomorphism groups, Topology 11 (1972), 1-44.

2. W. Browder, Torsion in H-spaces, Ann. of Math. (2) 74 (1961), 27-51.

3. P. F. Conner and E. R. Floyd, Fibring within a cobordism class, Michigan Math. J. 12 (1965), 33-47.

4. C. T. C. Wall, Determination of the corbodism ring, Ann. of Math. (2) 72 (1960), 292-311.

Department of Mathematical Sciences, Hunter College, New York, New York 10021 